\title{
Negacionismo y conflicto social
}

Denialism and social conflict

Miguel Moreno Muñoz

Profesor Titular, Departamento de Filosofía II, Universidad de Granada

\author{
ATEÍSMO, CREENCIA Y SENTIDO. HOMENAJE A JUAN ANTONIO ESTRADA \\ MONOGRÁFICO COORDINADO POR REMEDIOS ÁVILA Y MIGUEL MORENO
}

\section{RESUMEN}

Los enfoques negacionistas han adquirido un protagonismo destacable en el debate público. Aquí se analizan tres aspectos del fenómeno: 1) su carácter transversal en cuanto a núcleos de creencias e intereses originarios, que atraen fácilmente la atención por distanciarse de lo convencional; 2) la subestimación institucional del riesgo asociado, una vez constatada su capacidad para movilizar y captar adeptos con adscripciones ideológicas muy heterogéneas; y 3) algunos aspectos en los que el debate académico puede contribuir a la amplificación del fenómeno, explotando de manera poco afortunada las cautelas epistémicas relativas a criterios de demarcación, consenso especializado y metodologías de investigación interdisciplinar. Entre otros resultados, se sugiere prestar atención a las formas de negacionismo práctico que, por su convergencia con los objetivos e intereses del teórico, pueden generar dinámicas incontrolables de irracionalidad y conflicto social.

\section{ABSTRACT}

Denialist approaches have become prominent in public debate. This paper emphasizes three aspects of the phenomenon: 1) its cross-cutting nature in terms of core beliefs and original interests, which easily attract attention by distancing themselves from the conventional; 2) the institutional underestimation of the associated risk, once its capacity to mobilize and capture adherents with very heterogeneous ideological ascriptions has been ascertained; and 3) some aspects in which academic debate can contribute to the amplification of the phenomenon, exploiting in an unfortunate way epistemic caveats regarding demarcation criteria, specialized consensus and interdisciplinary research methodologies. Among other findings, it is suggested to pay more attention to multiple forms of practical denialism that remain outside critical scrutiny but, because of their convergence with the goals and interests of the theoretical, can trigger uncontrollable dynamics of irrationality and social conflict.

PALABRAS CLAVE

negacionismo | percepción pública | irracionalidad | conflicto social | epistemología del riesgo

KEYWORDS

denialism | public perception | irrationality | social conflict | epistemology of risk

\section{Introducción}

Los periodos de crisis social contribuyen a dar mayor visibilidad a planteamientos negacionistas en el debate público. Tales contextos lastran la toma de decisiones con múltiples restricciones de raíz económica, que de modo directo o indirecto generan dinámicas de exclusión y conflicto social. Imponen restricciones que evidencian las carencias democráticas de esquemas de funcionamiento interpretados como tecnocráticos en periodos de normalidad, pero cuestionados cuando fracasan en la distribución de costes ligados a los procesos de reajuste y medidas de adaptación o racionalidad económica, supuestamente respaldadas por conocimiento experto (Sager 2018).

Cuando en el dominio social del que se forma parte resultan inviables los proyectos vitales e intereses genuinos de colectivos numerosos, parece inevitable arrojar la sospecha sobre cualquier segmento del entramado político e institucional implicado en la toma de decisiones difíciles y atribuir el resultado a algún esquema de conspiración organizada (Stojanov y otros 2021, Ceballos 2021). Una situación que sistemáticamente se reproduce en circunstancias adversas de salud pública, tras episodios catastróficos de diversa naturaleza o en periodos de crisis global como la producida entre 2008 y 2012 (la Gran Recesión, según expresión de Stein 2012 y Stiglitz 2009).

En las sociedades avanzadas, estas circunstancias anómalas parecen reproducirse con mayor frecuencia por una combinación desafortunada de factores internos: inestabilidad política, auge de los movimientos identitarios, corrupción en diversas instancias administrativas o arribismo político (entre otros señalados por Ware 2011: 89-112 y 133-152), y externos: escasez de recursos y materias primas, 
conflictos geopolíticos, cambio climático, desarrollo tecnológico y globalización (estudiados por Trubowitz 1993, Hotez 2018, Coppola 2020 y Nance 2017).

El desfase entre la complejidad de los problemas que es preciso abordar y las limitaciones en los procedimientos para la toma de decisiones o gestión social del riesgo desencadena con frecuencia cursos de acción subóptimos, ampliamente cuestionados tanto en sus objetivos y modos de implementarse como en la eficacia y credibilidad de los actores involucrados (Kunreuther y Heal 2012, Hudson 2018). Si el escenario de crisis se prolonga, contribuye a minar la confianza en la cascada de información e indicadores de partida y la credibilidad epistémica de los actores e instituciones implicados en cada eslabón de la toma de decisiones (Roeser y otros 2012: 34, Huang y otros 2013).

Este proceso complejo de deslegitimación puede afectar a las instituciones, asociaciones científicas y colectivos profesionales involucrados en aspectos parciales, pero decisivos, de posibles soluciones como el desarrollo de vacunas y su administración a la población diana en situaciones de pandemia (Bayas 2011, Leiserowitz y otros 2013). Dinámicas previas de conflicto y polarización social pueden amplificar el alcance de la reacción social y extender su carácter transversal, con efectos impredecibles en la gestión de situaciones acuciantes (Kerr y otros 2021).

El objetivo de este trabajo es mostrar la distorsión que los planteamientos negacionistas introducen en la identificación de escenarios de crisis y en la justificación de los cursos de acción compatibles con el conocimiento disponible. Se asume que el colectivo de expertos y profesionales más conscientes de los riesgos subestima, por regla general, la capacidad de los grupos negacionistas para sobreexplotar la incertidumbre, sembrar la duda incluso sobre escenarios altamente probables y normalizar conductas de riesgo que terminan impactando en la población más vulnerable (Roeser y otros 2012: 229-230, Vulpe y Rughiniş 2021).

Aquí se analizan tres aspectos del fenómeno: 1) su carácter transversal en cuanto a núcleos de creencias e intereses originarios, que atraen fácilmente la atención por distanciarse de lo convencional; 2) la subestimación institucional del riesgo asociado, una vez constatada su capacidad para movilizar y captar adeptos con adscripciones ideológicas muy heterogéneas; y 3) algunos aspectos en los que el debate académico puede contribuir a la amplificación del fenómeno, explotando de manera poco afortunada las cautelas epistémicas relativas a criterios de demarcación, consenso especializado y metodologías de investigación interdisciplinar. Entre otros resultados, se sugiere prestar atención a las formas de negacionismo práctico que, por su convergencia con los objetivos e intereses del teórico, pueden generar dinámicas incontrolables de irracionalidad y conflicto social.

\section{Alcance y consecuencias del negacionismo}

El negacionismo científico suele focalizarse en dominios de problemas que se supone descifrados en sus elementos esenciales y bien conocidos entre la comunidad experta o explicados por una teoría científica específica. Ejemplos comunes son el negacionismo del holocausto (Rhead 2020), del sida (Diethelm y McKee 2008), de la utilidad de las vacunas (Vulpe y Rughiniş 2021) o de la asociación entre exposición al humo del tabaco y enfermedad pulmonar (Oreskes y Conway 2010: 136-168). Su reaparición fuera del ámbito cultural anglosajón resulta especialmente llamativa ante la consistencia de la evidencia acumulada durante décadas en campos disciplinares bien consolidados (Björnberg, y otros 2017) y su limitada visibilidad mediática en contexto europeo (Vesa y otros 2020).

Versiones más recientes, con adeptos entre los grupos de ideología menos conservadora e individualista, se centran en promover esquemas alternativos para evaluar los peligros de los organismos transgénicos (Roeser y otros 2012: 33 y 490-494), de las estelas (chemtrails) de los aviones a reacción (Mazon y otros 2017); la relación entre vacunas y casos de autismo (Toth 2020: 3-4) o el daño para la salud de los dispositivos inalámbricos y la tecnología 5G (Bräscher y otros 2017, Gallastegi y otros 2019).

Si en la cuestión ambiental el negacionismo alcanza ya una escala de financiación que Riley Dunlap considera típica de una industria de difusión cultural globalizada (Dunlap y McCright 2011: ch. 2.8) no debe extrañar la proyección de un patrón similar de distorsión del debate público sobre problemas de salud pública (Kata 2010), integración de minorías (Willen 2012) o estadísticas de violencia de género (UNWOMEN 2021, Canto y otros 2020, Fábián 2017, UN Report 2009). 
El creacionismo, la astrología, el Feng shui o las teorías sobre astronautas que visitaron la Tierra en el pasado pueden parecer memes culturales inocuos para la dinámica de sociedades alfabetizadas, con acceso generalizado a la educación obligatoria y programas que facilitan a casi el veinte por ciento de su población acceder a la educación superior. Pero en las cuestiones ambientales y de salud pública los planteamientos y campañas negacionistas pueden tener efectos devastadores para colectivos vulnerables de cualquier lugar, constatados entre enfermos de sida, población asentada en zonas de riesgo de inundación, trabajadores en industrias contaminantes y enfermos de cáncer, por mencionar ejemplos bien estudiados (Duffy 2005, London y Schneider 2012, Murray y otros 2006, Steinberg 2009).

El negacionismo del cambio climático aglutina a los partidarios de movimientos políticos conservadores e individualistas, y el negacionismo del holocausto u otros casos de genocidio vincula sobre todo a grupos de extrema derecha (Hansson 2017: 43-45, Valdivielso 2016). Pero sus mismas estrategias de cherrypicking en relación con la evidencia disponible, menosprecio del conocimiento interdisciplinar bien consolidado y prolongación artificial de controversias o debates ya superados pueden constatarse entre grupos que promueven el recurso a terapias alternativas (seudoterapias resulta más apropiado) y el descrédito de las instituciones científicas o gubernamentales responsables de gestionar escenarios complejos de riesgo (Björnberg y otros 2017, Hansson 2017: 41-44).

Derivado del anterior, el negacionismo de la extinción (extinction denialism) y del declive de la biodiversidad es otra incorporación reciente al catálogo de distorsiones que circulan por las redes sociales (Lees y otros 2020). Su objetivo no es otro que atenuar o neutralizar el impacto en la opinión pública de informes que ponen de manifiesto la gravedad de la crisis ecológica —con tendencias alarmantes, según los detalles que la agencia France-Presse ha filtrado del $6^{\circ}$ informe del IPCC - y la amenaza que supone tanto para especies emblemáticas de aves y mamíferos como para otras de las que depende el equilibrio de los ecosistemas (Brondizio y otros 2019, WWF 2020, AFP 2021, Maclean y Wilson 2011).

La ignorancia deliberada de información relevante para comprender la gravedad de la crisis ecológica y la escala de las transformaciones requeridas para mitigar el impacto de la actividad humana sobre los ecosistemas amenazados legitima a muchos actores privados o estatales para exigir la continuidad de actividades ambientalmente insostenibles, además de obstaculizar la adopción de acuerdos y medidas con el alcance requerido para aportar soluciones eficaces (Oreskes y Conway 2010: 10-35, Dunlop y Spratt 2018 y 2019, Painter y Kundzewicz 2018).

Los mensajes negacionistas y sus estrategias de difusión -que incluyen bulos, teorías de la conspiración y criterios alternativos de validación teórica para captar la atención y ganar adeptos en redes socialespueden inducir comportamientos irracionales (Ceballos 2021, Jolley y otros 2019); y distorsiones en la percepción de riesgo con la que operan porcentajes importantes de la población (Funk y Gramlich 2021). Estados Unidos, Brasil, la India y Reino Unido ilustran el potencial de los grupos negacionistas para dificultar extraordinariamente la gestión de la pandemia de COVID-19 bajo criterios de prevención científicamente fundados (Agiesta 2021, Abutaleb y otros 2020, Lopes 2021, Machado Silva 2021, Fonseca y otros 2021, Booth y Duncan 2020).

\section{Ideología, rechazo de la ciencia y promoción de seudoteorías}

La conducta de los grupos con escasa alfabetización científica resulta más influenciable por creencias sin fundamento cuando estas son asumidas y promovidas por líderes con cuya visión del mundo y esquemas de organización social sintonizan (Chigwedere y otros 2008). Esta dinámica se constata sobre todo entre los grupos con ideología conservadora y de derechas, pero también entre movimientos libertarios o sistemáticamente reacios al sometimiento de la voluntad individual a la norma estatal. Y responde a un patrón observado en temas tan heterogéneos como el cambio climático, la teoría de la evolución, los programas de vacunación y la seguridad de los organismos transgénicos (Lewandowsky y Oberauer 2021: 2-3).

Aunque en ciertos grupos las actitudes hacia los temas mencionados resultan condicionadas por convicciones religiosas o afinidades patrióticas y nacionalistas que les predisponen negativamente hacia los enfoques universalistas ineludibles en problemas de alcance global, la inconsistencia epistémica se agrava cuando tales convicciones incluyen el excepcionalismo humano para comprender los problemas ambientales y justificar el lugar preeminente de la especie humana en su relación con la naturaleza 
Esta base epistémica opera por igual incitando al rechazo de políticas ambientales orientadas a proteger la salud de la población en zonas vulnerables al impacto del cambio climático -cuando su consecución no es posible sin limitar actividades dañinas para el medio ambiente o declarando la protección de espacios ricos en biodiversidad- que instando la prohibición de toda investigación dirigida al estudio de enfermedades de base genética, si requiere la manipulación de embriones humanos en alguna fase de su desarrollo.

Los procesos motivacionales subyacentes parecen condicionar de modo similar las prioridades políticas de individuos vinculados a grupos de izquierda y de quienes se identifican con el espectro liberalconservador o de derechas (Washburn y Skitka 2018). Sin embargo, el rechazo de la evidencia científica y la disposición a priorizar las referencias ideológicas, religiosas o morales como base para articular la comprensión personal y actitudes hacia las vacunas, el cambio climático o la investigación biomédica caracteriza en mayor medida a los grupos de la derecha política e ideológica de la última década (Lewandowsky y Oberauer 2021: 2).

A esto han contribuido campañas como la desarrollada por la industria tabaquera para contrarrestar la pérdida de negocio esperable si prosperaban las demandas en los tribunales que aportaban resultados de investigación compatibles con una asociación fuerte entre exposición al humo del tabaco y desarrollo de enfermedad pulmonar grave (Oreskes y Conway 2010: 59-65). Personajes como Frederick Seitz, William Nierenberg y Fred Singer trabajaron incansablemente para convencer al público estadounidense de que todo el establishment científico estaba controlado por científicos liberales y activistas de ideología comunista, infiltrados en las principales agencias gubernamentales (Oreskes y Conway 2010: 63-64).

Pero tales estrategias, diseñadas para generar confusión y sobreexplotar la duda, no impidieron que grupos conservadores y progresistas de Estados Unidos y otros países desarrollados se pusieran de acuerdo en impulsar programas ambiciosos de financiación de la investigación científica durante la segunda mitad del siglo XX, con resultados tan exitosos como el Protocolo de Montreal para prevenir la destrucción de la capa de ozono (Oreskes y Conway 2010: 125) o la erradicación de la viruela (CAV 2020).

La diferencia hoy probablemente esté menos en la sutileza y capacidad financiera de los nuevos impulsores de contra-narrativas negacionistas que en los incentivos para instrumentalizar la desconfianza hacia las instituciones científicas y actores gubernamentales, con la finalidad de consolidar nuevas mayorías y desarrollar políticas ultraconservadoras en cuestiones ambientales, energéticas, salud pública e integración de minorías (Plehwe 2014).

El núcleo de creencias compartido por los opositores a los programas de vacunación apenas ha evolucionado desde finales del siglo XIX. Incluye la exageración o invención de efectos adversos, cuya ocultación se produce de forma deliberada; la finalidad de lucro de la industria farmacéutica, reforzada mediante actores estatales corruptos; ingredientes que combinan productos químicos peligrosos; más probabilidad de daño que de beneficio; despotismo y totalitarismo orwelliano ligado a los programas de salud pública; e insistencia en la eficacia de estilos de vida saludable como solución alternativa (Wolfe y Sharp 2002: 325 y 460).

Por desgracia, tampoco han variado sus consecuencias: litigios en los tribunales que retrasan la adopción de medidas eficaces de prevención; capacidad para movilizar a un porcentaje considerable de la población en situaciones difíciles de gestionar para las autoridades sanitarias; episodios con brotes importantes de las enfermedades que se pretende erradicar; y pérdida evitable de miles de vidas entre grupos vulnerables (Altman 2020, Lipton y otros 2021, Schneider 2020, Malta y otros 2021, McPhillips y Gan 2021).

Si en el siglo XIX los principales cauces de difusión eran artículos de prensa, cartas, libros, revistas y panfletos advirtiendo de los peligros de la vacunación, Internet y los servicios en línea de las grandes plataformas tecnológicas han multiplicado los canales (aplicaciones de mensajería instantánea; blogs, prensa electrónica y foros; agregadores de noticias; servidores de podcasts y vídeos) con capacidad para llegar a una audiencia mundial y dominar importantes nichos socioculturales. La consolidación de nuevas pautas de interacción social a través de redes y plataformas tecnológicas, en ecosistemas de servicios financiados mediante publicidad segmentada, amplifica bulos y mensajes negacionistas en tanto contribuyen a mantener activos durante el mayor tiempo posible a millones de usuarios interesados 
(Moreno 2018, Cinelli y otros 2021, Mclntyre 2018: 17-34 y 89-122).

En medios gestionados por profesionales del periodismo no son infrecuentes prácticas que, bajo el criterio de escuchar a todas las partes implicadas en un problema (Mclntyre 2018: 84), airean mensajes negacionistas en horarios de máxima audiencia y otorgan similar espacio o reconocimiento tanto a expertos profesionalmente acreditados como a defensores de teorías de la conspiración y promotores de seudoteorías (Serrano 2021, Meneses-Fernández y Santana-Hernández 2020, Liu y otros 2020).

Como complemento a los canales de mayor visibilidad y atracción para grupos diana, los actores negacionistas desarrollan desde hace décadas una intensa actividad de cabildeo (lobbying) e infiltración institucional, con el objetivo de sesgar a su favor el debate sobre los marcos reguladores y retrasar o impedir la aprobación de propuestas legislativas contrarias a sus intereses en los ámbitos institucionales competentes (Almiron y otros 2020, Plehwe 2014). Entre sus objetivos de especial interés figuran instituciones académicas de referencia, sobre todo las que gestionan determinadas publicaciones periódicas o colecciones editoriales destacadas (Sherrington 2021, Robaina 2020).

La pérdida de credibilidad de los medios tradicionales ha ido ligada al desplazamiento de los contratos publicitarios hacia plataformas que aseguran mayor audiencia y cuotas de atención entre millones de usuarios, vinculados a ecosistemas de servicios que apenas rinden cuentas por la veracidad o calidad de los contenidos (Del Vicario y otros 2016). La pérdida de ingresos por publicidad se ha intentado compensar en muchos casos ofreciendo solo contenidos por suscripción, lo cual excluye a grandes colectivos de lectores potenciales con bajo poder adquisitivo del acceso a contenidos generados por profesionales de la comunicación mínimamente comprometidos con las exigencias de veracidad, equilibrio y responsabilidad informativa.

El resultado es una mayor visibilidad de medios con pocos escrúpulos y el reforzamiento de actores con fuertes incentivos para amplificar cascadas de rumores, seudoteorías e invención de noticias o conspiraciones delirantes (Ceballos 2021: caps. 2, 9 y 11). La intervención de bots y servicios de redireccionado automático termina amplificando contenidos que acaparan la atención de sus destinatarios porque explotan sesgos bien conocidos en el modo de focalizar la atención y la memoria sobre contenidos falsos en relación con la política, la salud, el medio ambiente, la amenaza terrorista o crisis y desastres de todo tipo (Vosoughi y otros 2018, Mclntyre 2018: 43-62). Bastan porcentajes moderados de influencia en la población para desencadenar episodios impredecibles de irracionalidad colectiva, más o menos graves en función de las circunstancias (Nature, ed. 2021, Burkle 2020b y 2020a).

\section{Negacionismo práctico}

A nadie sorprende que el ánimo de lucro pueda sesgar el juicio individual en la elección de cursos de acción en el ámbito privado o en la representación de cualquier aspecto de la realidad social sujeto a incertidumbre. La falta de cautela en consumidores que eligen productos de escasa utilidad y alto coste siguiendo las recomendaciones automáticas en ciertas plataformas, por pereza para contrastar ofertas y comprobar sus características, no parece reprobable mientras el coste de la decisión no recaiga sobre terceros. Dependiendo del volumen de afectados, un sistema de recomendaciones automáticas que sesga la elección hacia productos de escasa utilidad y alto coste probablemente llame pronto la atención de alguna instancia reguladora en materia de consumo y acumule quejas suficientes para sustanciar un caso de mala praxis en el diseño o funcionamiento de la plataforma.

Pero en actividades como la oferta de servicios sanitarios y la venta de productos de uso médico importan las cautelas epistémicas y los criterios teóricos o experimentales para demarcar entre tratamientos de eficacia probada y seudoterapias. En mayor medida cuando se reducen los recursos disponibles y cabe anticipar escenarios de mayor demanda. Imprudencias por falta de cautela epistémica aceptables en el ámbito privado pueden constituir ejemplos de mala praxis y negligencia cuando se producen en contextos de actividad profesional. La diferencia estriba en la cualificación teórica que se presupone a los actores acreditados para el ejercicio de determinadas actividades y las garantías exigidas por el marco regulador para adecuar su actuación a la lex artis (es decir, al conocimiento fiable disponible), reduciendo al mínimo la probabilidad de acierto por error o accidente (Pritchard 2014: 12-17 y 46-52). 
La dinámica social incluye muchas formas de cooperación mediadas por actores, instituciones y prácticas dependientes de investigación especializada para generar conocimiento, identificar las mejores prácticas y adecuar los procesos de cualificación. A quienes pasan el filtro se les presupone ciertas capacidades psicológicas, aptitudes y competencias cognitivas. Una parte sustancial de los recursos humanos y materiales se destina a la detección del fraude, del intrusismo profesional y de prácticas subóptimas o negligentes. Pero mientras la libertad de cátedra permite en las aulas universitarias someter a examen incluso los planteamientos teóricos más descabellados, y se valora la actitud crítica ante creencias que durante siglos no pudieron ser cuestionadas, no ocurre lo mismo con las prácticas que promueven creencias irracionales, inducen a error y pueden ocasionar pérdida de vidas humanas, daños patrimoniales y deterioro del medio natural. Quienes las promueven introducen grietas importantes en la comunidad epistémica y contribuyen a distorsionar las disposiciones (capacidades psicológicas, competencias cognitivas, aptitudes y habilidades) que permiten distinguir el conocimiento de la superstición y la irracionalidad en contextos relevantes (Sosa 2018: 10).

En su dimensión práctica, el negacionismo opera sobredimensionando el alcance de los elementos de incertidumbre, generando ruido sobre correlaciones bien estudiadas y distorsionando a la baja la percepción de riesgo sobre cursos de acción que la evidencia y el análisis teórico desaconsejan. En particular, cuando se trata de procesos cuya materialización temporal ocurre de forma gradual e involucra dinámicas de prueba y estudio en múltiples dominios profesionales. Así, se presenta como escepticismo genuinamente científico una suerte de nihilismo epistémico extremo, sin límites reconocibles porque utiliza cualquier resquicio de incertidumbre para equiparar las opiniones de expertos y legos, susceptibles por igual al conflicto de intereses y con idéntica probabilidad de incurrir en sesgos cognitivos (Coady y Corry 2013: 14).

Como estrategia retórica, el encuadre negacionista se despliega minando en la práctica cualquier criterio de demarcación, desprestigiando a las instituciones y colectivos profesionales que han contribuido al desarrollo y estandarización de las metodologías de investigación capaces de proporcionar evidencia científica interdisciplinar y reduciendo a mera opinión lastrada por ideología toda atribución de responsabilidad. Puede constatarse con un patrón reconocible en casos de accidente laboral por falta de medidas de seguridad, en tragedias derivadas de la manipulación de residuos peligrosos o en procesos complejos de genocidio y violaciones graves de los derechos humanos. Bajo este enfoque, todo intento de aportar evidencia del daño causado e identificar a las víctimas usando procedimientos y metodologías estandarizadas es considerado una obsesión epistémica más propia de distopías orwellianas que de esfuerzos genuinos por comprender el mundo y evitar tergiversaciones de los poderes dominantes (Oreskes y Conway 2010: 236-237).

A diferencia del escepticismo teórico y práctico que promueven los actores negacionistas, quienes trabajan en disciplinas consolidadas se entrenan para desarrollar la disposición a no creer ninguna afirmación (incluidos los enunciados nucleares de las teorías científicas) carente de respaldo mediante pruebas o argumentos válidos (evidencia). Pero, además, dominan estilos de razonamiento y procedimientos técnicos (experimentación, observación, medida, simulación matemática, modelización computacional de sistemas complejos) que contribuyen a ampliar y refinar el conocimiento previo (Gramelsberger 2011: 296-298). Entre sus aptitudes y habilidades cognitivas se presupone la capacidad para ajustar su actuación a un criterio operativo y explicable de evidencia suficiente (la demostración de que ciertas afirmaciones tienen más probabilidades de ser verdaderas que de no serlo, sobre el trasfondo de conocimiento previo bien establecido) y de manejar ciertas cautelas en la proyección de sus modelos sobre escenarios sujetos a incertidumbre (Odenbaugh 2018, Knutti 2018, Rougier y Crucifix 2018).

La combinación de estas competencias en diverso grado les cualifica profesionalmente y, en ciertos casos, como personas expertas cuyo criterio informado e imparcial resulta imprescindible para comprender aspectos técnicamente complejos en un proceso probatorio, evitando los sesgos en el testimonio de las partes interesadas. Se trata de un mecanismo institucionalizado de cooperación social al que se destinan recursos importantes, para garantizar en lo posible que la atribución de responsabilidad se sustente en evidencia robusta y no en mero azar o habilidad retórica (McCain 2016: 237-251).

\section{Negacionismo y debate político}

En mucho contextos, los procesos de toma de decisiones priorizan la conveniencia política en detrimento 
del conocimiento experto y presionan para controlar los encuadres informativos en los medios de mayor difusión. Las distorsiones en la percepción de riesgo que induce el encuadre negacionista se agravan cuando son aireadas por líderes responsables de la toma de decisiones, con capacidad para movilizar a miles de partidarios. Pueden resultar letales en episodios de alerta por altas temperaturas, epidemias, riesgo de inundaciones e incendios, donde son cruciales los tiempos de respuesta (Lidskog y Sundqvist 2013, Moreno 2021). Pero también en la gestión de escenarios de crisis de gestación lenta y gradual, como señalan Stiglitz a propósito del libre mercado y la falta de regulación que agravó la Gran Recesión (2010), Pritchard (2014: 90-91) en relación con la politización del fenómeno migratorio o Stern (2016: 408) en relación con el impacto del cambio climático.

De la calidad de la información proporcionada depende la eficacia de las estrategias preventivas y de los recursos -sanitarios, emergencias, seguridad y protección civil- movilizados, en particular si la población es alertada a tiempo y comprende la gravedad del riesgo al que se enfrenta. Un marco de gestión social del riesgo sistemáticamente escorado hacia el lado menos dramático y con incentivos políticos o económicos para enfatizar los márgenes más conservadores de las proyecciones contribuye a consolidar percepciones escépticas y actitudes imprudentes ante las amenazas (Molina y otros 2018, Moreno 2019), que agravan su impacto y contribuyen a generar escenarios de crisis y conflicto social eventualmente inmanejables (Aven y otros 2011: 1075-1076).

El episodio de asalto al congreso de Estados Unidos por una turba enardecida con las arengas de su presidente ha puesto en el foco del debate público la importancia que revisten los esquemas de liderazgo como factor de riesgo en sí mismos (Lewis 2018). Pero las advertencias sobre el impacto de campañas electorales sustentadas en la difusión continua de bulos y opiniones disparatadas para ganar visibilidad ya se habían multiplicado tras la campaña en favor del Brexit y el fortalecimiento de los movimientos de extrema derecha en Europa (Mclntyre 2018: 152-172).

Difícilmente pueden atribuirse a quienes llegan al poder con una campaña de bulos y seudoteorías de la conspiración las disposiciones epistémicas indispensables para gestionar con responsabilidad episodios de crisis prolongada. Menos aún si su gestión involucra áreas de actividad y problemas bajo el radar de los think tanks negacionistas. El menosprecio de la evidencia y del conocimiento experto en la gestión de asuntos cotidianos tiene consecuencias, pues compromete aspectos críticos en la dinámica de decisiones individuales y colectivas que aglutina las acciones necesarias para afrontar problemas de mayor calado en situaciones de crisis económica, desastres naturales o pandemia (Lewis 2021).

A toda retórica de la confrontación como estrategia para ganar visibilidad o respaldo político subyace alguna versión del negacionismo práctico. En ambas se da prioridad al componente emocional sobre la evidencia, en detrimento de la reflexión juiciosa sobre aspectos complejos de la realidad social a la que el oponente podría contribuir. El resultado es un clima de desconfianza hacia todos los actores con capacidad de alcanzar puestos de decisión y el menosprecio de las instancias capaces de aportar criterio experto, consideradas tan susceptibles al sesgo y la tergiversación como el votante común. La distorsión del debate público sirve de indicador sobre el aumento de la entropía y el riesgo de conflicto social, en la medida que obstaculiza cualquier intento de alinear los intereses individuales con las iniciativas para afrontar los desafíos colectivos más apremiantes (Davie 2014).

\section{Conclusión}

La relación entre negacionismo, promoción de seudoteorías y conflicto social se ha visto reforzada por la convergencia de estudios que analizaron el fenómeno como una variante espuria y socialmente dañina del escepticismo filosófico (Bunge 2002: 181-246); como un problema de demarcación (Hansson 2009 y 2017); como efecto de mecanismos psicocognitivos de autoengaño y autolegitimación ideológica (Fasce 2017, Lewandowsky y Oberauer 2021); y como estrategia organizada para ofuscar la evidencia sobre el cambio climático antropogénico y el consenso acerca de actividades dañinas para la salud o el medio natural (Oreskes y Conway 2010).

El debate sobre la dimensión tecnocrática de los mecanismos de gobernanza en las democracias liberales y su instrumentalización por las élites de la burocracia weberiana no parecía evolucionar gran cosa desde el análisis del fenómeno hecho por Frank Fischer (1990) y Ulrich Beck (2012). Entre las contribuciones que advertían del riesgo para la democracia de la resistencia populista a los expertos cabe destacar la de Bertsou y Caramani (2020). Pero fueron las campañas electorales de 2016 y el 
escándalo de Cambridge Analytica los detonantes para articular nuevos enfoques del fenómeno como estrategia de confrontación social y acceso al poder, en el contexto de cambios tecnológicos inducidos por el auge de las grandes plataformas de servicios digitales y el declive de los medios tradicionales (Vosoughi y otros 2018, Moreno 2018a y 2018b).

En tanto que fenómeno multidimensional y complejo para los esquemas convencionales de gestión del riesgo, el cambio climático había captado la atención de instituciones involucradas en el análisis prospectivo de amenazas para la seguridad nacional, considerando en particular los riegos asociados a las cadenas de suministro de alimentos, desplazamientos masivos de población (IOM 2009) y la mayor probabilidad de nuevas enfermedades y plagas (Barnosky y otros 2012, Battisti y Naylor 2009, Behnassi y Yaya 2011). Pero su encuadre como riesgo existencial y vector de conflictos de gran alcance parecía un encuadre minoritario (Burke y otros 2015, Coates 2009) hasta que estudios más recientes y exhaustivos enfatizaron la gravedad de los impactos y las interpretaciones excesivamente conservadoras que habían caracterizado a las estimaciones de riesgo previas (Dangendorf y otros 2017, Guha-Sapir y Hoyois 2015, Whitmee y otros 2015).

Mi contribución amplía el enfoque de autores como Dunlop y Spratt (2018 y 2019), quienes señalan el coste de la inacción y del retraso en la adopción de medidas de prevención, adaptación y mitigación al cambio climático como factor de desestabilización mundial y acelerador de otros ciclos de crisis humanitaria y socio-política. Sostengo que las narrativas y estrategias negacionistas se difunden con extraordinaria eficacia -en comparación con los procesos generales de alfabetización y sensibilizaciónen un escenario complejo de posibilidades técnicas y desafíos a una escala sin precedentes, debilitando los mecanismos de cooperación social en todos los niveles, minando la credibilidad de actores e instituciones responsables de la toma de decisiones e incentivando dinámicas de irracionalidad colectiva en la gestión social del riesgo.

La probabilidad de que actores y grupos negacionistas tomen el control de las mayorías parlamentarias -y de las instituciones responsables de aportar informes especializados sobre cuestiones ambientales, de salud o escenarios verosímiles de crisis- constituye en sí mismo un factor de riesgo y desestabilización, constatada su capacidad para distorsionar el debate público, movilizar recursos en la dirección equivocada y agravar dinámicas previas de conflictos sin resolver. La pandemia ha proporcionado ejemplos dramáticos de las consecuencias esperables de una gestión del riesgo en manos de actores que alardean de sus convicciones negacionistas y desprecian el criterio experto. También de la ineficacia y arbitrariedad de líderes políticos que no se distancian expresamente del consenso científico en sus planteamientos teóricos pero avalan múltiples formas de negacionismo práctico en cuestiones de salud, seguridad laboral y política ambiental.

\section{Notas}

Esta contribución forma parte de un volumen monográfico en homenaje al profesor Juan Antonio Estrada Díaz, catedrático emérito de la Universidad de Granada, a quien agradezco su disposición incansable a contrastar ideas y someter a escrutinio público sus creencias y convicciones intelectuales, en ámbitos muy propensos a la intolerancia y al dogmatismo.

\section{Bibliografía}

Abutaleb, Y. (y otros)

2020 "The U.S. was beset by denial and dysfunction as the coronavirus raged", The Washington Post, 4 abril. https://www.washingtonpost.com/national-security/2020/04/04/coronavirus-government-dysfunction/ 
Agiesta, J.

2021 "CNN Poll: About a quarter of adults say they won't try to get a Covid-19 vaccine",

https://edition.cnn.com/2021/04/29/politics/cnn-poll-covid-vaccines/index.html

Almiron, N. (y otros)

2020 "Dominant counter-frames in influential climate contrarian European think tanks", Climatic Change, no 162 (4): 2003-2020. https://doi.org/10.1007/s10584-020-02820-4.

Altman, D.

2020 "Understanding the US failure on coronavirus-an essay by Drew Altman", BMJ: m3417. https://doi.org/10.1136/bmj.m3417.

Aven, T. (y otros)

2011 "On the ontological status of the concept of risk", Safety Science, $\mathrm{n}^{\circ} 49$ (8-9): 1074-1079. https://doi.org/10.1016/j.ssci.2011.04.015.

Barnosky, A. D. (y otros)

2012 "Approaching a state shift in Earth/'s biosphere", Nature, nº 486 (7401): 52-58.

https://doi.org/10.1038/nature11018.

Battisti, D. S. (y R. L. Naylor)

2009 "Historical Warnings of Future Food Insecurity with Unprecedented Seasonal Heat", Science, $\mathrm{n}^{\circ} 323$ (5911): 240-244. https://doi.org/10.1126/science.1164363.

Bayas, J. M.

2011 "Talking about vaccines: The scientific point of view. Consensus document of the Scientific Societies and Associations against the anti-vaccine campaign", Vacunas, $n^{\circ} 12(4): 160-161$.

https://doi.org/10.1016/S1576-9887(11)70025-5.

Beck, U. (y M. Chalmers)

1998 "Risk Society and the Provident State", en S. Lash y otros (ed.), Risk, Environment and Modernity: Towards a New Ecology, pp. 28-43. London, SAGE Publications Ltd.

https://doi.org/10.4135/9781446221983.n2.

Behnassi, M. (y S. Yaya)

2011 "Food Crisis Mitigation: The Need for an Enhanced Global Food Governance", en M. Behnassi, S. Draggan y S. Yaya (ed.), Global Food Insecurity. Springer, Dordrecht: 93-125. https://doi.org/10.1007 1978-94-007-0890-7_8.

Bertsou, E. (y D. Caramani)

2020 The Technocratic Challenge to Democracy. London New York, Routledge, Taylor y Francis Group.

Björnberg, K. E. (y otros)

2017 "Climate and environmental science denial: A review of the scientific literature published in 1990-2015", Journal of Cleaner Production, $\mathrm{n}^{\circ}$ 167: 229-241. https://doi.org/10.1016 /j.jclepro.2017.08.066.

Booth, R. (y P. Duncan)

2020 "Nearly 1,500 Deaths in One Day: UK Ministers Accused of Downplaying Covid-19 Peak", The Guardian, 19 de junio.

https://www.theguardian.com/world/2020/jun/19/over-1000-deaths-day-uk-ministers-accused-

downplaying-covid-19-peak

Bräscher, A.-K. (y otros)

2017 "Are media reports able to cause somatic symptoms attributed to WiFi radiation? An experimental test of the negative expectation hypothesis", Environmental Research, no 156: 265-271. https://doi.org /10.1016/j.envres.2017.03.040.

Brondizio, E. S. (y otros)

2019 IPBES. 2019. Global assessment report on biodiversity and ecosystem services of the Intergovernmental Science- Policy Platform on Biodiversity and Ecosystem Services, 31 de mayo. Bonn, IPBES Secretariat. https://www.ipbes.net/global-assessment-biodiversity-ecosystem-services 
Bunge, $\mathrm{M}$.

2002 Crisis y reconstrucción de la filosofía. Barcelona, Gedisa.

Burke, M. (y otros)

2015 "Climate and Conflict", Annual Review of Economics, $\mathrm{n}^{\circ} 7$ (1): 577-617.

https://doi.org/10.1146/annurev-economics-080614-115430.

Burkle, F. M.

2020a "Declining Public Health Protections within Autocratic Regimes: Impact on Global Public Health Security, Infectious Disease Outbreaks, Epidemics, and Pandemics", Prehospital and Disaster Medicine, $\mathrm{n}^{\circ} 35$ (3): 237-246. https://doi.org/10.1017/S1049023X20000424.

2020 b "Political Intrusions into the International Health Regulations Treaty and Its Impact on Management of Rapidly Emerging Zoonotic Pandemics: What History Tells Us", Prehospital and Disaster Medicine, $\mathrm{n}^{\circ}$ 35 (4): 426-430. https://doi.org/10.1017/S1049023X20000515.

Canto, J. M. (y otros)

2020 "The Influence of Ideological Variables in the Denial of Violence Against Women: The Role of Sexism and Social Dominance Orientation in the Spanish Context", International Journal of Environmental Research and Public Health, no 17 (14): 4934. https://doi.org/10.3390/ijerph17144934.

CAV (Comité Asesor de Vacunas)

2020 "Hace 40 años desde la erradicación de la viruela", 14 de mayo.

https://vacunasaep.org/profesionales/noticias/hace-40-anos-desde-la-erradicacion-de-la-viruela

Ceballos, N.

2021 El pensamiento conspiranoico. Barcelona, Arpa.

Chigwedere, P. (y otros)

2008 "Estimating the Lost Benefits of Antiretroviral Drug Use in South Africa", JAIDS - Journal of Acquired Immune Deficiency Syndromes, $\mathrm{n}^{\circ} 49$ (4): 410-415. https://doi.org/10.1097 /QAI.0b013e31818a6cd5.

Cinelli, M. (y otros)

2021 "The echo chamber effect on social media", Proceedings of the National Academy of Sciences, $\mathrm{n}^{\circ}$ 118 (9): e2023301118. https://doi.org/10.1073/pnas.2023301118.

Coady, D. (y R. Corry)

2013 The climate change debate: an epistemic and ethical enquiry. Houndmills, Basingstoke, Hampshire New York, NY, Palgrave Macmillan.

Coates, J. F.

2009 "Risks and threats to civilization, humankind, and the earth", Futures, no 41 (10): 694-705. https://doi.org/10.1016/j.futures.2009.07.010.

Coppola, D. P.

2020 "Hazards" en E. Coppola (ed.), Introduction to International Disaster Management. Amsterdam, Butterworth-Heinemann: cap. 2, pp. 47-176. https://doi.org/10.1016/B978-0-12-817368-8.00002-6.

Dangendorf, S. (y otros)

2017 "Reassessment of 20th century global mean sea level rise", Proceedings of the National Academy of Sciences, $n^{\circ} 114$ (23): 5946-5951. https://doi.org/10.1073/pnas.1616007114.

Davie, N.

2014 "A Less than Polite People? Incivility, Ruffianism and Anti-Social Behaviour in Urban England, 1830-1900", en Anti-social Behaviour in Britain, pp. 3-17. https://doi.org/10.1057/9781137399311_1.

Del Vicario, M. (y otros)

2016 "The spreading of misinformation online", Proceedings of the National Academy of Sciences, $\mathrm{n}^{\circ} 113$ (3): 554-559. https://doi.org/10.1073/pnas.1517441113.

Diethelm, P. (y M. McKee)

2008 "Denialism: what is it and how should scientists respond?", The European Journal of Public Health, 
n 19 (1): 2-4. https://doi.org/10.1093/eurpub/ckn139.

Duffy, L.

2005 "Suffering, shame, and silence: The stigma of HIVIAIDS", Journal of the Association of Nurses in AIDS Care, no 16 (1): 13-20. https://doi.org/10.1016/j.jana.2004.11.002.

Dunlap, R. E. (y A. M. McCright)

2011 "Organized Climate Change Denial" en J. S. Dryzek, R. B. Norgaard y D. Schlosberg (ed.), The Oxford Handbook of Climate Change and Society. Oxford University Press.

https://doi.org/10.1093/oxfordhb/9780199566600.003.0010.

Dunlop, D. (y I. Spratt)

2018 "What Lies Beneath: The Understatement of Existential Climate Risk". Breakthrough - National Centre for Climate Restoration. http://climateextremes.org.au/wp-content/uploads/2018/08/What-LiesBeneath-V3-LR-Blank5b15d.pdf

Ecklund, E. H. (y otros)

2017 "Examining Links Between Religion, Evolution Views, and Climate Change Skepticism", Environment and Behavior, $\mathrm{n}^{\circ} 49$ (9): 985-1006. https://doi.org/10.1177/0013916516674246.

Fábián, K.

2017 "The Politics of Domestic Violence in Central Europe: International and Domestic Contestations" en Global Responses to Domestic Violence. Springer International Publishing: 125-149. https://doi.org /10.1007/978-3-319-56721-1_7.

Fasce, A.

2017 "Los parásitos de la ciencia. Una caracterización psicocognitiva del engaño pseudocientífico", THEORIA. An International Journal for Theory, History and Foundations of Science, $\mathrm{n}^{\circ} 32$ (3): 347. https://doi.org/10.1387/theoria.17775.

Finlay, B. L. (y A. D. Workman)

2013 "Human exceptionalism", Trends in Cognitive Sciences, $\mathrm{n}^{\circ} 17$ (5): 199-201.

https://doi.org/10.1016/j.tics.2013.03.001.

Fischer, F.

1990 Technocracy and the Politics of Expertise. Newbury Park/London/New Delhi, Sage.

Fonseca, E. M. (y otros)

2021 "Political discourse, denialism and leadership failure in Brazil's response to COVID-19", Global Public Health: 1-16. https://doi.org/10.1080/17441692.2021.1945123

Funk, C. (y J. Gramlich)

2021 "10 facts about Americans and coronavirus vaccines", 23 de marzo. Pew Research Center. https://pewrsr.ch/393DxfV

Gallastegi, M. (y otros)

2019 "Exposure and health risks perception of extremely low frequency and radiofrequency electromagnetic fields and the effect of providing information", Environmental Research, $\mathrm{n}^{\circ} 169:$ 501-509. https://doi.org/10.1016/j.envres.2018.11.042.

Gramelsberger, G.

2011 "What do numerical (climate) models really represent?" Studies in History and Philosophy of Science" Part A, nº 42 (2): 296-302. https://doi.org/10.1016/j.shpsa.2010.11.037.

Guha-Sapir, D. (y P. Hoyois)

2015 "Estimating populations affected by disasters: A review of methodological issues and research gaps". Centre for Research on the Epidemiology of Disasters (CRED), Institute of Health and Society (IRSS), Brussels: 1-15. https://www.preventionweb.net/go/45775

Hansson, S. O.

2009 "Cutting the Gordian Knot of Demarcation", International Studies in the Philosophy of Science, $\mathrm{n}^{\circ} 23$

(3): 237-243. https://doi.org/10.1080/02698590903196007. 
2017 "Science denial as a form of pseudoscience", Studies in History and Philosophy of Science, Part A, n 63: 39-47. https://doi.org/10.1016/j.shpsa.2017.05.002.

Hotez, P. J.

2018 "Human Parasitology and Parasitic Diseases: Heading Towards 2050", in D. Rollinson y J. R. Stothard (ed.), Advances in Parasitology. Academic Press, vol. 100, cap. 2: 29-38.

https://doi.org/10.1016/bs.apar.2018.03.002.

Huang, L. (y otros)

2013 "The influence of public perception on risk acceptance of the chemical industry and the assistance for risk communication", Safety Science, $n^{\circ} 51$ (1): 232-240.

https://doi.org/10.1016/j.ssci.2012.05.018.

Hudson, M.

2018 "Ending technocracy with a neologism? Avivocracy as a conceptual tool", Technology in Society, $\mathrm{n}^{\circ}$ 55: 136-139. https://doi.org/10.1016/j.techsoc.2018.07.006.

IOM

2009 Migration, environment and climate change: assessing the evidence, F. Laczko y C. Aghazarm (ed.). International Organization for Migration (IOM).

https://publications.iom.int/system/files/pdf/migration_and_environment.pdf

Jolley, D. (y otros)

2019 "Belief in conspiracy theories and intentions to engage in everyday crime", British Journal of Social Psychology, $\mathrm{n}^{\circ} 58$ (3): 534-549. https://doi.org/10.1111/bjso.12311.

Kata, A.

2010 "A postmodern Pandora's box: Anti-vaccination misinformation on the Internet", Vaccine, $\mathrm{n}^{\circ} 28$ (7): 1709-1716. https://doi.org/10.1016/j.vaccine.2009.12.022.

Kerr, J. (y otros)

2021 "Political polarization on COVID-19 pandemic response in the United States", Personality and Individual Differences, $\mathrm{n}^{\circ}$ 179: 110892. https://doi.org/10.1016/j.paid.2021.110892.

Knutti, R.

2018 "Climate Model Confirmation: From Philosophy to Predicting Climate in the Real World", en Climate Modelling: Philosophical and Conceptual Issues. Springer International Publishing, pp. 325-359. https://doi.org/10.1007/978-3-319-65058-6_11.

Kunreuther, H. (y G. Heal)

2012 Managing Catastrophic Risk. National Bureau of Economic Research, Cambridge, M. A. https://www.nber.org/system/files/working_papers/w18136/w18136.pdf

Lees, A. C. (y otros)

2020 "Biodiversity scientists must fight the creeping rise of extinction denial", Nature Ecology \& Evolution, no 4 (11): 1440-1443. https://doi.org/10.1038/s41559-020-01285-z.

Leiserowitz, A. A.

2013 "Climategate, Public Opinion, and the Loss of Trust", American Behavioral Scientist, $\mathrm{n}^{\circ} 57$ (6): 818-837. https://doi.org/10.1177/0002764212458272.

Lewandowsky, S. (y K. Oberauer)

2021 "Worldview-motivated rejection of science and the norms of science", Cognition, $\mathrm{n}^{\circ} 215: 104820$. https://doi.org/10.1016/j.cognition.2021.104820.

Lewis, M.

2018 The fifth risk: undoing democracy. Londres, Allen Lane-Penguin Books.

2021 The premonition: a pandemic story. Londres, Allen Lane.

Lidskog, R. (y G. Sundqvist)

2013 "Sociology of Risk", In S. Roeser y otros (ed.), Essentials of Risk Theory. Springer, Dordrecht: 75-105. https://doi.org/10.1007/978-94-007-5455-3_4. 
Lipton, E. (y otros)

2021 "He Could Have Seen What Was Coming: Behind Trump's Failure on the Virus", The New York

Times, 11/04/2020. https://www.nytimes.com/2020/04/11/us/politics/coronavirus-trump-response.html

Liu, F. H. M. (y otros)

2020 "Contrasting communications of sustainability science in the media coverage of palm oil agriculture on tropical peatlands in Indonesia, Malaysia and Singapore", Environmental Science \& Policy, $\mathrm{n}^{\circ} 114$ : 162-169. https://doi.org/10.1016/j.envsci.2020.07.004.

London, L. (y H. Schneider)

2012 "Globalisation and health inequalities: Can a human rights paradigm create space for civil society action?", Social Science \& Medicine, n 74 (1): 6-13. https://doi.org/10.1016/j.socscimed.2011.03.022.

Lopes, M. F.

2021 "From denial to hope: Brazil deals with a prolonged COVID-19 epidemic course", Nature Immunology, $\mathrm{n}^{\circ} 22$ (3): 256-257. https://doi.org/10.1038/s41590-021-00875-8.

Machado, $\mathrm{H}$.

2021 "The Brazilian Scientific Denialism Through The American Journal of Medicine", The American Journal of Medicine, $\mathrm{n}^{\circ} 134$ (4): 415-416. https://doi.org/10.1016/j.amjmed.2021.01.003.

Maclean, I. M. D. (y R. J. Wilson)

2011 "Recent ecological responses to climate change support predictions of high extinction risk", Proceedings of the National Academy of Sciences, $n^{\circ} 108$ (30): 12337-12342.

https://doi.org/10.1073/pnas.1017352108.

Malta, M. (y otros)

2021 "Political neglect of COVID-19 and the public health consequences in Brazil: The high costs of science denial", EClinicalMedicine, n 35: 100878. https://doi.org/10.1016/j.eclinm.2021.100878.

Mazon, J. (y otros)

2017 "Airplane clouds: From chemtrail pseudoscience to the science of contrails", Mètode Revista de Difusió de La Investigació, nº 8. https://doi.org/10.7203/metode.8.9954.

McCain, K.

2016 The Nature of Scientific Knowledge. Springer International Publishing, https://doi.org/10.1007 1978-3-319-33405-9.

McIntyre, L.

2018 Post-truth. Cambridge, MA, The MIT Press.

McPhillips, D. (y N. Gan)

2021 "Global Covid-19 death toll surpasses 4 million", CNN.Com, 8/07/2021.

https://edition.cnn.com/2021/07/07/world/global-covid-death-toll-4-million-intl-hnk/index.html

Meneses-Fernández, M. D. (y J. D. Santana-Hernández)

2020 "Construcción periodística de la mala praxis científica: análisis en diarios digitales", Revista Prisma Social, n 31: 40-63. https://revistaprismasocial.es/article/view/3853.

Molina, M. (y otros)

2018 "Climate report understates threat", The Bulletin of the Atomic Scientists, 9/10/2021. https://thebulletin.org/2018/10/climate-report-understates-threat

Moreno, M.

2018a "Mediación tecnológica de la interacción social y riesgos de su instrumentalización: el caso de la plataforma Facebook", Gazeta de Antropología, $n^{\circ} 34$ (2), art. 03. http://www.gazeta-antropologia.es $/ ? \mathrm{p}=5084$

2018b "Virtualización del espacio público y concepto débil de privacidad. Lecciones del caso FacebookCambridge Analytica", Ensayos de Filosofia, $\mathrm{n}^{\circ} 8$ (2/3).

http://www.ensayos-filosofia.es/archivos/articulo/virtualizacion-del-espacio-publico-y-concepto-debil-deprivacidad-lecciones-del-caso-facebook-cambridge-analytica

2019 "La subestimación del riesgo catastrófico como sesgo epistemológico", Ensayos de Filosofía, n 9 
(1). http://www.ensayos-filosofia.es/archivos/articulo/subestimar-el-riesgo-catastrofico

2021 "El coste de la distorsión informativa en situaciones de alerta por fenómenos potencialmente catastróficos", en R. Mancinas-Chávez y M. L. Cárdenas-Rica (ed.), Medios y comunicación en tiempos de posverdad. Madrid, Fragua: 274-291. https://doi.org/10.5281/zenodo.4619876.

Murray, E. J. (y otros)

2006 "Emerging health issues: the widening challenge for population health promotion", Health Promotion International, $\mathrm{n}^{\circ} 21$, supl. 1 (4): 15-24. https://doi.org/10.1093/heapro/dal047.

Nance, M. T. (y W. A. Boettcher)

2017 "Conflict, cooperation, and change in the politics of energy interdependence: An introduction", Energy Research \& Social Science, $\mathrm{n}^{\circ}$ 24: 1-5. https://doi.org/10.1016/j.erss.2016.12.020.

Nature (Editorial)

2021 "The world must learn from COVID before diving into a pandemic treaty", Nature, $\mathrm{n}^{\circ} 592$ (7853): 165-166. https://doi.org/10.1038/d41586-021-00866-7.

Odenbaugh, J.

2018 "Building Trust, Removing Doubt? Robustness Analysis and Climate Modeling", en A. L. Elisabeth y E. Winsberg (ed.), Climate Modelling. Springer International Publishing: 297-321.

https://doi.org/10.1007/978-3-319-65058-6_10.

Oreskes, N. (y E. M. Conway)

2010 Merchants of Doubt: How a Handful of Scientists Obscured the Truth on Issues from Tobacco Smoke to Global Warming. New York, Bloomsbury Press.

Painter, J. (y Z. W. Kundzewicz)

2018 "COP24 in coal country: why Poland is Europe's climate denial capital", 10 de diciembre. The Conversation, https://theconversation.com/cop24-in-coal-country-why-poland-is-europes-climate-denialcapital-103573

Plehwe, D.

2014 "Think tank networks and the knowledge-interest nexus: the case of climate change", Critical Policy Studies, no 8 (1): 101-115. https://doi.org/10.1080/19460171.2014.883859.

Pritchard, D.

2014 What is this thing called knowledge (Third). New York, Routledge /Taylor and Francis.

Rhead, J. C.

2020 "Modern Holocaust", Explore, no 16 (1): 69-70. https://doi.org/10.1016/j.explore.2019.11.004.

Robaina, E.

2020 "Los ocho 'think tanks' negacionistas del clima más importantes de Europa”, Climática - La marea.com, 30 de septiembre.

https://www.climatica.lamarea.com/think-tanks-negacionistas-europa/

Roeser, S. (y otros)

2012 Handbook of Risk Theory. https://doi.org/10.1007/978-94-007-1433-5.

Rougier, J. (y M. Crucifix)

2018 "Uncertainty in Climate Science and Climate Policy", en A. L. Elisabeth y E. Winsberg (ed.), Climate Modelling. Springer International Publishing: 361-380. https://doi.org/10.1007/978-3-319-65058-6_12.

Sager, F.

2018 "Policy evaluation and democracy: Do they fit?", Evaluation and Program Planning, n 69: 125-129. https://doi.org/10.1016/j.evalprogplan.2017.08.004.

Schneider, E. C.

2020 "Failing the Test - The Tragic Data Gap Undermining the U.S. Pandemic Response", New England Journal of Medicine, $\mathrm{n}^{\circ} 383$ (4): 299-302. https://doi.org/10.1056/NEJMp2014836.

Serrano, $\mathrm{P}$. 
2021 “¿Había que entrevistar a Miguel Bosé?”, ElDiario.es, 14 de abril.

https://www.eldiario.es/opinion/zona-critica/habia-entrevistar-miguel-bose_129_7799668.html

Sherrington, R.

2021 "Oxford University Professor Condemned for 'Legitimising Lies and Falsehoods' of Climate Denial Group", Desmog.com (19 de mayo), https://www.desmog.com/2021/05/19/oxford-university-professorclimate-fossil-fuels/

Sosa, E.

2018 Conocimiento reflexivo, vol. II. Zaragoza, Prensas de la Universidad de Zaragoza (introducción, traducción y notas de Manuel Liz).

Spratt, D. (y I. Dunlop)

2019 Existential climate-related security risk: A scenario approach. Melbourne, Breakthrough - National Centre for Climate Restoration.

https://docs.wixstatic.com/ugd/148cb0_a1406e0143ac4c469196d3003bc1e687.pdf

Stein, $\mathrm{H}$.

2012 "The neoliberal policy paradigm and the great recession", Panoeconomicus, $\mathrm{n}^{\circ} 59$ (4): 421-440. https://doi.org/10.2298/PAN1204421S.

Steinberg, J.

2009 "The AIDS denialists", New Scientist, n 202 (2713): 32-36.

https://doi.org/ 10.1016/S0262-4079(09)61658-4.

Stern, N.

2016 "Economics: Current climate models are grossly misleading", Nature, nº 530 (7591): 407-409. https://doi.org/10.1038/530407a

Stiglitz, J. E.

2009 Interpreting the causes of the Great Recession of 2008. Lecture at BIS Conf., Basel, June 2009: 1-23. https://fcic-static.law.stanford.edu/cdn_media/fcic-testimony/2009-1020-Stiglitz-article-2.pdf 2010 Free Fall: America, Free Markets and the Sinking of the World Economy. New York, Norton.

Stojanov, A. (y otros)

2021 "Examining a domain-specific link between perceived control and conspiracy beliefs: a brief report in the context of COVID-19", Current Psychology, June 2021. https://doi.org/10.1007 /s12144-021-01977-0.

Toth, C.

2020 "To Vaccinate or Not to Vaccinate My Child? - What Is at Stake in Vaccination Repertoires?", On Education. Journal for Research and Debate, n 3 (8). https://doi.org/10.17899/on_ed.2020.8.8.

Trubowitz, P.

1993 "Political conflict and foreign policy in the United States", Political Geography, no 12 (2): 121-135. https://doi.org/10.1016/0962-6298(93)90032-3.

UN Report

2009 "15 years of the United Nations special rapporteur on violence against women, its causes and consequences", https://www.ohchr.org/Documents/Issues/Women/15YearReviewofVAWMandate.pdf

\section{UNWOMEN}

2021 "Global Database on Violence against Women", https://evaw-global-database.unwomen.org/en. Síntesis en: https://www.unwomen.org/en/what-we-do/ending-violence-against-women/facts-and-figures.

Valdivielso, J.

2016 "La negación del desafío ecológico: cómo se destiñe el verde”, en Á. Valencia Sáiz y R. E. Aguilera Portales (ed.), Democracia verde. Ciudad de México, Editorial Porrúa: 259-280.

Vesa, J. (y otros)

2020 "The quiet opposition: How the pro-economy lobby influences climate policy", Global Environmental Change, $n^{\circ}$ 63: 102117. https://doi.org/10.1016/j.gloenvcha.2020.102117. 
Vosoughi, S. (y otros)

2018 "The spread of true and false news online", Science, $\mathrm{n}^{\circ} 359$ (6380): 1146-1151.

https://doi.org/10.1126/science.aap9559

Vulpe, S.-N. (y C. Rughiniş)

2021 "Social amplification of risk and "probable vaccine damage": A typology of vaccination beliefs in 28 European countries", Vaccine, no 39 (10): 1508-1515. https://doi.org/10.1016/j.vaccine.2021.01.063

Ware, A.

2011 Political Conflict in America. New York, Palgrave Macmillan US.

https://doi.org/10.1057/9781137010339.

Washburn, A. N. (y L. J. Skitka)

2018 "Science Denial Across the Political Divide: Liberals and Conservatives Are Similarly Motivated to Deny Attitude-Inconsistent Science", Social Psychological and Personality Science, $\mathrm{n}^{\circ} 9$ (8): 972-980. https://doi.org/10.1177/1948550617731500.

Whitmee, S. (y otros)

2015 "Safeguarding human health in the Anthropocene epoch: report of The Rockefeller FoundationLancet Commission on planetary health", The Lancet, $\mathrm{n}^{\circ} 386$ (10007): 1973-2028.

https://doi.org/10.1016/S0140-6736(15)60901-1.

Willen, S. S.

2012 "Migration, "illegality," and health: Mapping embodied vulnerability and debating health-related deservingness", Social Science and Medicine, $\mathrm{n}^{\circ} 74$ (6): 805-811.

https://doi.org/10.1016/j.socscimed.2011.10.041.

Wolfe, R. M. (y L. K. Sharp)

2002 "Anti-vaccinationists past and present", BMJ, nº 325 (7361): 430-432.

https://doi.org/10.1136/bmj.325.7361.430.

WWF

2020 Living Planet report 2020 - Bending the curve of biodiversity loss, R.E.A. Almond, M. Grooten y T. Petersen (ed.), Gland, Switzerland. https://livingplanet.panda.org/en-gb/ 